\title{
Reactive oxygen species mediate high glucose-induced heparanase-1 production and heparan sulphate proteoglycan degradation in human and rat endothelial cells: a potential role in the pathogenesis of atherosclerosis
}

\author{
G. Rao $・$ H. G. Ding $\cdot$ W. Huang $\cdot$ D. Le $・$ \\ J. B. Maxhimer • A. Oosterhof • T. van Kuppevelt • \\ H. Lum • E. J. Lewis • V. Reddy • R. A. Prinz • X. Xu
}

Received: 22 October 2010 /Accepted: 1 February 2011 / Published online: 20 March 2011

(C) Springer-Verlag 2011

\begin{abstract}
Aims/hypothesis The content of heparan sulphate is reduced in the endothelium under hyperglycaemic conditions and may contribute to the pathogenesis of atherosclerosis. Heparanase-1 (HPR1) specifically degrades heparan sulphate proteoglycans. We therefore sought to determine whether: (1) heparan sulphate reduction in endothelial cells is due to increased HPR1 production through increased reactive oxygen species (ROS) production; and (2) HPR1 production is increased in vivo in endothelial cells under hyperglycaemic and/or atherosclerotic conditions.

Methods HPR1 mRNA and protein levels in endothelial cells were analysed by RT-PCR and Western blot or HPR1 enzymatic activity assay, respectively. Cell surface heparan
\end{abstract}

G. Rao $\cdot$ H. G. Ding $\cdot$ W. Huang $\cdot$ D. Le $\cdot$ J. B. Maxhimer $\cdot$

R. A. Prinz $\cdot X . X u(\square)$

Department of General Surgery, Rush University Medical Center,

1653 W. Congress Parkway,

Chicago, IL 60612, USA

e-mail: xxu@rush.edu

A. Oosterhof · T. van Kuppevelt

Department of Biochemistry, Nijmegen Centre for Molecular

Life Sciences, Radboud University Nijmegen Medical Centre,

Nijmegen, the Netherlands

H. Lum

Department of Pharmacology, Rush University Medical Centre,

Chicago, IL, USA

\section{E. J. Lewis}

Department of Internal Medicine,

Rush University Medical Centre,

Chicago, IL, USA sulphate levels were analysed by FACS. HPR1 in the artery from control rats and a rat model of diabetes, and from patients under hyperglycaemic and/or atherosclerotic conditions was immunohistochemically examined.

Results High-glucose-induced HPR1 production and heparan sulphate degradation in three human endothelial cell lines, both of which were blocked by ROS scavengers, glutathione and $N$-acetylcysteine. Exogenous $\mathrm{H}_{2} \mathrm{O}_{2}$ induced HPR1 production, subsequently leading to decreased cell surface heparan sulphate levels. HPR1 content was significantly increased in endothelial cells in the arterial walls of a rat model of diabetes. Clinical studies revealed that HPR1 production was increased in endothelial cells under hyperglycaemic conditions, and in endothelial cells and macrophages in atherosclerotic lesions.

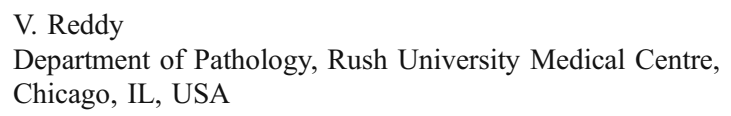

Present address:

J. B. Maxhimer

Department of Surgery,

The Johns Hopkins University School of Medicine, Baltimore, MD, USA

Present address:

R. A. Prinz

Department of Surgery, NorthShore University HealthSystem,

Evanston, IL, USA 
Conclusions/interpretation Hyperglycaemia induces HPR1 production and heparan sulphate degradation in endothelial cells through ROS. HPR1 production is increased in endothelial cells from a rat model of diabetes, and in macrophages in the atherosclerotic lesions of diabetic and non-diabetic patients. Increased HPR1 production may contribute to the pathogenesis and progression of atherosclerosis.

Keywords Atherosclerosis - Diabetes · Endothelial cells . Heparanase $\cdot$ Heparan sulphate $\cdot$ Hyperglycaemia

Macrophage $\cdot$ Reactive oxygen species

$\begin{array}{ll}\text { Abbreviations } \\ \text { HBMEC } & \text { Human brain microvascular endothelial cells } \\ \text { HDMEC } & \text { Human dermal microendothelial cells } \\ \text { HPR1 } & \text { Heparanase-1 } \\ \text { HSPG } & \text { Heparan sulphate proteoglycans } \\ \text { NAC } & N \text {-acetylcysteine } \\ \text { ROS } & \text { Reactive oxygen species } \\ \text { VSV } & \text { Vesicular stomatitis virus }\end{array}$

\section{Introduction}

Heparan sulphate proteoglycans (HSPG) are important components of the cell surface, the extracellular matrix and the basement membrane. HSPG comprise a protein core that is covalently attached to a unique glycosaminoglycan chain characterised by a linear array of alternating disaccharide units [1-4]. HSPG play an important role in the assembly and structure of the basement membrane, the regulation of basement membrane permeability, and in growth factor activity and cellular adhesion [1, 2, 5-7]. Emerging evidence indicates that the amounts of HSPG are reduced under hyperglycaemic conditions in endothelial cells in vitro $[8,9]$ and in vivo in diabetic patients and animal models of disease [10]. A significant decrease in heparan sulphate, but a relative increase in dermatan sulphate in normal and atherosclerotic intima of diabetic patients precede the development of lesions in diabetes [10]. Loss of arterial heparan sulphate correlates with the onset of atherosclerosis in a monkey model of diabetes [11] and accelerates the deposition of lipoprotein(a) in the subendothelial matrix [12-15]. The mechanisms of hyperglycaemia-induced heparan sulphate decrease in endothelial cells remain poorly understood.

Heparanase-1 (HPR1) is an endoglycosidase that specifically degrades HSPG. Recent studies have shown that HPR1 production is increased in renal epithelial cells under hyperglycaemic conditions, subsequently leading to increased heparan sulphate degradation [16-18]. In a rat model of adriamycin nephropathy, reactive oxygen species (ROS) production is required for induction of HPR1 production in glomerular epithelial cells [19]. Increased HPR1 production has been observed in endothelial cells under high glucose conditions in vitro [20]. The notion of whether ROS production is required for high glucose-induced HPR1 production and heparan sulphate degradation in endothelial cells remains to be tested.

\section{Methods}

Reagents and cell lines $N$-acetylcysteine (NAC) and glutathione were purchased from Sigma (St Louis, MO, USA). 2,7-Dichlorodihydrofluorescin diacetate was purchased from Molecular Probes (Eugene, OR, USA). Heparin sodium salt (H4784) was purchased from Sigma. PI-88 was kindly provided by Progen Pharmaceuticals (Toowong, QLD, Australia). Human brain microvascular endothelial cells (HBMEC) and human dermal microendothelial cells (HDMEC) were used in our previous study [21]. HBMEC were grown in RPMI 1640 containing 10\% FBS, MEM vitamins, non-essential amino acid, sodium pyruvate (Invitrogen, Carlsbad, CA, USA), penicillin (100 U/ml), streptomycin $(100 \mu \mathrm{g} / \mathrm{ml})$ and $2 \mathrm{mmol} / \mathrm{l}$ L-glutamine. HDMEC were grown in MCDB-131 medium (Invitrogen, Carlsbad, CA, USA) containing 5\% FBS (vol./vol.), $1 \mathrm{mg} / \mathrm{ml}$ hydrocortisone, penicillin $(100 \mathrm{U} / \mathrm{ml})$, streptomycin $(100 \mu \mathrm{g} / \mathrm{ml})$ and $2 \mathrm{mmol} / \mathrm{l} \mathrm{L}$-glutamine. EaHy 926 cell line was purchased from the American Tissue Culture Collection (Manassas, VA, USA) and cells grown in DMEM. A rabbit anti-HPR1 polyclonal IgG was used in Western blot to detect HPR1 in the conditioned medium (H-80, sc-25825; Santa Cruz, San Diego, CA, USA). AntiHPR1 monoclonal antibody and polyclonal antibody were kindly provided by $\mathrm{H}$. Miao (ImClone Systems, New York, NY, USA). The specificity of the antibodies has been previously described and verified in our Western blot using purified HPR1 and the cell lysates of HPR1transfected cells [22]. Anti-vesicular stomatitis virus (VSV) tag monoclonal antibody (clone P5D4) was purchased from Sigma. Alexa Fluor 488-conjugated goatanti-mouse $\operatorname{IgG}$ antibody was from Invitrogen. Antiheparan sulphate monoclonal antibody (clone HepSS) was purchased from Seikagaku (Chuo-ku, Tokyo, Japan). Phage display-derived anti-heparan sulphate antibody with a VSV tag (EW3D10) has been previously described [23].

HPR1 activity assay Endothelial cells grown for $48 \mathrm{~h}$ in the media containing 5 or $30 \mathrm{mmol} / \mathrm{l}$ glucose were collected and analysed for HPR1 activity in cell lysates according to a novel ELISA protocol established in this laboratory [18, $22,24,25]$. 
Luciferase reporter gene expression HBMEC were transfected with the luciferase reporter gene driven by a 0.3 , 0.7 or $3.5 \mathrm{~kb} \mathrm{HPRl} \mathrm{(also} \mathrm{known} \mathrm{as} \mathrm{HPSE)} \mathrm{promoter}$ (pGL3/HPR1-0.3, pGL3/HPR1-0.7 and pGL3/HPR1-3.5) [26]. pCMV/SPORT (Invitrogen), which encodes a $\beta$ galactosidase gene, was co-transfected as an internal control. After incubation for $48 \mathrm{~h}$ in the medium containing 5 or $30 \mathrm{mmol} / \mathrm{l}$ glucose, the cells were collected and analysed for luciferase activity. The relative light unit in each sample was normalised against $\beta$ galactosidase activity measured by a colorimetric assay as previously reported [27]. The means of data in triplicate from one of at least two experiments with similar results are presented.

Flow cytometric analysis Cells were starved of glucose overnight and then incubated for $48 \mathrm{~h}$ in the medium containing 5 or $30 \mathrm{mmol} / \mathrm{l}$ glucose or the indicated concentrations of $\mathrm{H}_{2} \mathrm{O}_{2}$ in the absence or presence of three HPR1 inhibitors, PI-88, heparin and sulodexide (50 $\mu \mathrm{g} / \mathrm{ml}$ each) [28]. Cell surface heparan sulphate was stained with an anti-heparan sulphate monoclonal antibody (clone HepSS) according to previous publications [18]. To verify the specificity of high glucose-induced HPR1 production, HBMEC were seeded in a six well plate in complete RPMI 1640 medium. Upon 60\% confluence, cells were replenished with fresh medium containing different concentrations of glucose. Cell surface heparan sulphate levels were analysed by staining with an anti-heparan sulphate antibody (EW3D10) followed by a mouse anti-VSV monoclonal antibody and then by Alexa Fluor 488-conjugated goat anti-mouse IgG. For negative controls, normal mouse IgG was used to replace anti-VSV monoclonal antibody. To confirm the role of HPR1 in mediating high glucose-induced heparan sulphate degradation, HBMEC were transfected with a pcDNA3.1 vector (Invitrogen) or the vector encoding human HPR1 gene in sense (pcDNA/HPR1) or antisense orientation (pcDNA/HPRl-AS) [22] using FuGENE6 (Roche Applied Science, Indianapolis, IN, USA) transfection reagent. After incubation for $24 \mathrm{~h}$, the cells were treated with low or high glucose for $24 \mathrm{~h}$ and then analysed for cell surface heparan sulphate by flow cytometry of anti-heparan sulphate antibody (EW3D10)stained cells.

$R T-P C R$ HBMEC were treated for $24 \mathrm{~h}$ with 5 or $30 \mathrm{mmol} /$ 1 glucose, or with $5 \mathrm{mmol} / 1$ glucose in the presence of the indicated concentration of $\mathrm{H}_{2} \mathrm{O}_{2}$. HPRl mRNA was analysed by semi-quantitative RT-PCR as previously described $[18,29]$. In some experiments, HPR 1 transcription was analysed by real-time PCR according to our recent publication [30].
Western blot HPR1 in conditioned media and cell lysates was enriched by sepharose-heparin beads (Amersham Biosciences, Piscataway, NJ, USA) as previously published [31]. HPR1 protein in the supernatant fractions, presenting mainly as the $65 \mathrm{kDa}$ proenzyme, was detected by Western blot with a rabbit polyclonal antibody (Santa Cruz) that recognises the $65 \mathrm{kDa}$ form of HPR1. The majority of HPR1 protein in the cytosol, mainly in the lysosomes, is presented as the active enzyme, consisting of a $50 \mathrm{kDa}$ plus an $8 \mathrm{kDa}$ heterodimer [32, 33]. HPR1 in the cell lysates was detected by Western blot with an anti-HPR1 monoclonal antibody (ImClone) that recognises 50 and $65 \mathrm{kDa}$ forms of HPR 1.

Measurement of intracellular ROS HBMEC were seeded in six-well plates in the complete medium. Upon $50 \%$ confluence, the cells were starved of glucose overnight and then incubated in the medium containing 5 or $30 \mathrm{mmol} / 1$ glucose for the indicated lengths of time. Extra $25 \mathrm{mmol} / 1 \mathrm{~L}$-glucose was added to the cells under normal glucose conditions to maintain equal osmotic strength. Cells were collected and loaded with fluoroscein dye (2,7-dichlorodihydrofluorescin diacetate; $20 \mathrm{nmol} / \mathrm{l}$ ) for $15 \mathrm{~min}$ at $37^{\circ} \mathrm{C}$, followed by FACS analysis for intracellular ROS levels.

Induction of diabetes in rats Diabetes was induced in five Sprague-Dawley rats by a single dose, i.v. injection (55 mg/kg) of streptozotocin (Sigma). Long-acting insulin (Humulin; Eli Lilly, Indianapolis, IN, USA) was given daily to diabetic rats at a dose of $3 \mathrm{U}$ by subcutaneous injection to maintain glucose at about $27.75 \mathrm{mmol} / 1(500 \mathrm{mg} / \mathrm{dl})$. Control rats $(n=6)$ received the same volume of vehicle (0.1 mol/1 sodium citrate, $\mathrm{pH} 5.0)$. Body weight was recorded weekly. Blood glucose levels were measured using a kit (Ascensia Elite XL; Bayer, Elkhart, IN, USA). Normal and diabetic rats were killed on day 28. The aortic arteries were removed and fixed in formalin. Tissues were embedded within $24 \mathrm{~h}$ following a standard pathology procedure.

Patient information and tissue specimens Use of specimens from human patients was approved by the Institutional Review Board of Rush University Medical Center. The participants gave informed consent. Specimens $(n=10)$ from deceased patients (three carotid artery specimens from patients with diabetes, seven from patients without diabetes) were analysed for HPR1 levels (Table 1). Autopsy arterial specimens were taken within $30 \mathrm{~h}$ of death. Specimens $(n=15)$ of carotid atherosclerotic lesions from endarterectomy were fixed in formalin. Among them, six were from non-diabetic patients and nine from diabetic patients (Table 2). The samples were fixed in 10\% formalin (wt/vol.) and processed for embedding within $48 \mathrm{~h}$ follow- 
Table 1 HPR1 positivity in the autopsy specimens of carotid arteries

\begin{tabular}{|c|c|c|c|c|c|c|c|c|}
\hline \multicolumn{4}{|c|}{ Donor characteristics } & \multicolumn{3}{|c|}{ HPR1 levels ${ }^{\mathrm{a}}$} & \multicolumn{2}{|l|}{ Other donor variables } \\
\hline Number & Age & Sex & Diabetes & $\mathrm{EC}$ & Mac & $\mathrm{SMC}$ & Atherosclerosis $(\%)$ & Cause of death \\
\hline 1 & 46 & $\mathrm{~F}$ & No & + & - & - & Mild & Leukaemia and complications \\
\hline 2 & 34 & M & No & + & +++ & + & $30-50$ & Unknown \\
\hline 3 & 43 & $\mathrm{~F}$ & No & + & $\mathrm{N} / \mathrm{A}$ & +++ & Minimal & Small lung cell carcinoma \\
\hline 4 & 55 & M & No & + & +++ & +++ & 20 & Bladder carcinoma \\
\hline 5 & 23 & $\mathrm{~F}$ & No & + & N/A & +++ & Minimal & Pulmonary hypertension \\
\hline 6 & 62 & $\mathrm{~F}$ & No & ++ & N/A & +++ & $20-50$ & Myocardial infarction \\
\hline 7 & 81 & M & No & +++ & +++ & +++ & $75-100$ & Arterial disease \\
\hline 8 & 64 & M & Yes & +++ & +++ & +++ & 100 & Myocardial infarction + leukaemia \\
\hline 9 & 78 & M & Yes & +++ & ++ & - & $50-90$ & Small lung cell carcinoma \\
\hline 10 & 54 & M & Yes & +++ & +++ & +++ & 100 & Unknown \\
\hline
\end{tabular}

EC, endothelial cells; F, female; M, male; Mac, macrophages; N/A, not applicable; SMC, smooth muscle cells

${ }^{\text {a }}$ Graded as follows: - , no signal at all; + , weak signal in $>20 \%$ of cells; ++ , strong signal in more than $50 \%$ of cells; and +++ , strong signal in $>80 \%$ of cells

ing standard diagnostic pathology procedures. The sections of paraffin-embedded blocks were obtained from the Department of Pathology. Patient information is listed in Tables 1 and 2 .

Immunohistochemistry analysis of HPR 1 content The sections of paraffin-embedded arteries were analysed for HPR1 content by immunohistochemistry staining with a monoclonal antibody (ImClone) against HPR1 as previously described [25, 29, 34], except that antigen retrieval was conducted by microwave-heating the slides in $6 \mathrm{~mol} / \mathrm{l}$ urea for $30 \mathrm{~min}$. At least three sections of aortic artery from normal or diabetic rats were stained and graded. HPR1 levels were graded as: negative $(-)$, i.e. no signal at all; positive $(+)$, with weak signal in $>20 \%$ of cells; moderately positive $(++)$, with strong signal in $>50 \%$ of cells; and strongly positive $(+++)$, with strong signal in $>80 \%$ of cells. HPR1 levels were graded by two investigators (X. Xu and V. Reddy) in a blinded fashion. Endothelial cells, macrophages and smooth muscle cells in the artery were identified by an experienced pathologist (V. Reddy) who is specialised in cardiovascular pathology.

Statistical analysis Fisher's exact test was used to analyse the significance of a difference in HPR 1 positivity in the atherosclerotic plaques from diabetic and non-diabetic
Table 2 HPR1 positivity in the cells of carotid atherosclerotic plaques

EC, endothelial cells; F, female; $\mathrm{M}$, male; Mac, macrophages; N/A, not available due to absence of SMC; ND, not done; SMC, smooth muscle cells

${ }^{\text {a }}$ Graded as follows: -, no signal at all; + , weak signal in $>20 \%$ of cells; ++ , strong signal in more than $50 \%$ of cells; and +++ , strong signal in $>80 \%$ of cells

${ }^{\mathrm{b}}$ The values represent the range of lowest and highest blood glucose levels during hospitalization for surgery. In some patients, only a single measurement of blood glucose is available

\begin{tabular}{|c|c|c|c|c|c|c|c|c|}
\hline \multicolumn{4}{|c|}{ Donor characteristics } & \multicolumn{3}{|c|}{ HPR1 positivity ${ }^{\mathrm{a}}$} & \multicolumn{2}{|l|}{ Glycaemia } \\
\hline Number & Age & Sex & Diabetes & $\mathrm{EC}$ & Mac & $\mathrm{SMC}$ & Glucose $(\mathrm{mmol} / \mathrm{l})^{\mathrm{b}}$ & $\mathrm{HbA}_{1 \mathrm{c}}(\%)$ \\
\hline 1 & 77 & $\mathrm{~F}$ & No & - & ++ & N/A & $5.2-10.2$ & ND \\
\hline 2 & 71 & M & No & + & + & - & $5.9-10.9$ & ND \\
\hline 3 & 70 & $\mathrm{~F}$ & No & ++ & +++ & - & $6.1-8.6$ & 5.6 \\
\hline 4 & 74 & $\mathrm{~F}$ & No & ++ & + & - & $6.5-11.0$ & ND \\
\hline 5 & 65 & M & No & +++ & +++ & - & 4.35 & ND \\
\hline 6 & 80 & M & No & +++ & +++ & + & 6.55 & ND \\
\hline 7 & 82 & $\mathrm{~F}$ & Yes & - & + & - & $3.4-13.6$ & 6.7 \\
\hline 8 & 79 & M & Yes & - & + & N/A & 10.62 & 7.3 \\
\hline 9 & 61 & $\mathrm{~F}$ & Yes & + & ++ & - & $5.5-11.7$ & 6.3 \\
\hline 10 & 57 & M & Yes & ++ & +++ & ++ & 7.98 & 9.0 \\
\hline 11 & 66 & M & Yes & ++ & +++ & N/A & $7.0-13.3$ & ND \\
\hline 12 & 61 & $\mathrm{~F}$ & Yes & ++ & +++ &,+++ & $5.5-8.3$ & ND \\
\hline 13 & 79 & M & Yes & ++ & +++ & + & 5.12 & ND \\
\hline 14 & 67 & $\mathrm{~F}$ & Yes & +++ & +++ & ++ & $6.2-7.8$ & 10.4 \\
\hline 15 & 73 & M & Yes & +++ & +++ & +++ & 12.10 & 6.1 \\
\hline
\end{tabular}


patients. Unpaired Student's $t$ test was used to determine the significance of a difference of HPR1 activity (Figs 1a, 2d, 3e) or HPR1 mRNA levels (Figs 2c, 4a) between the control group and the experiment groups treated with high glucose or $\mathrm{H}_{2} \mathrm{O}_{2}$. Mann-Whitney $U$ test was used to determine a significant difference in HPR1 positivity in endothelial or smooth muscle cells in the arterial wall from normal and diabetic rats (Fig. 5e). A value of $p<0.05$ was

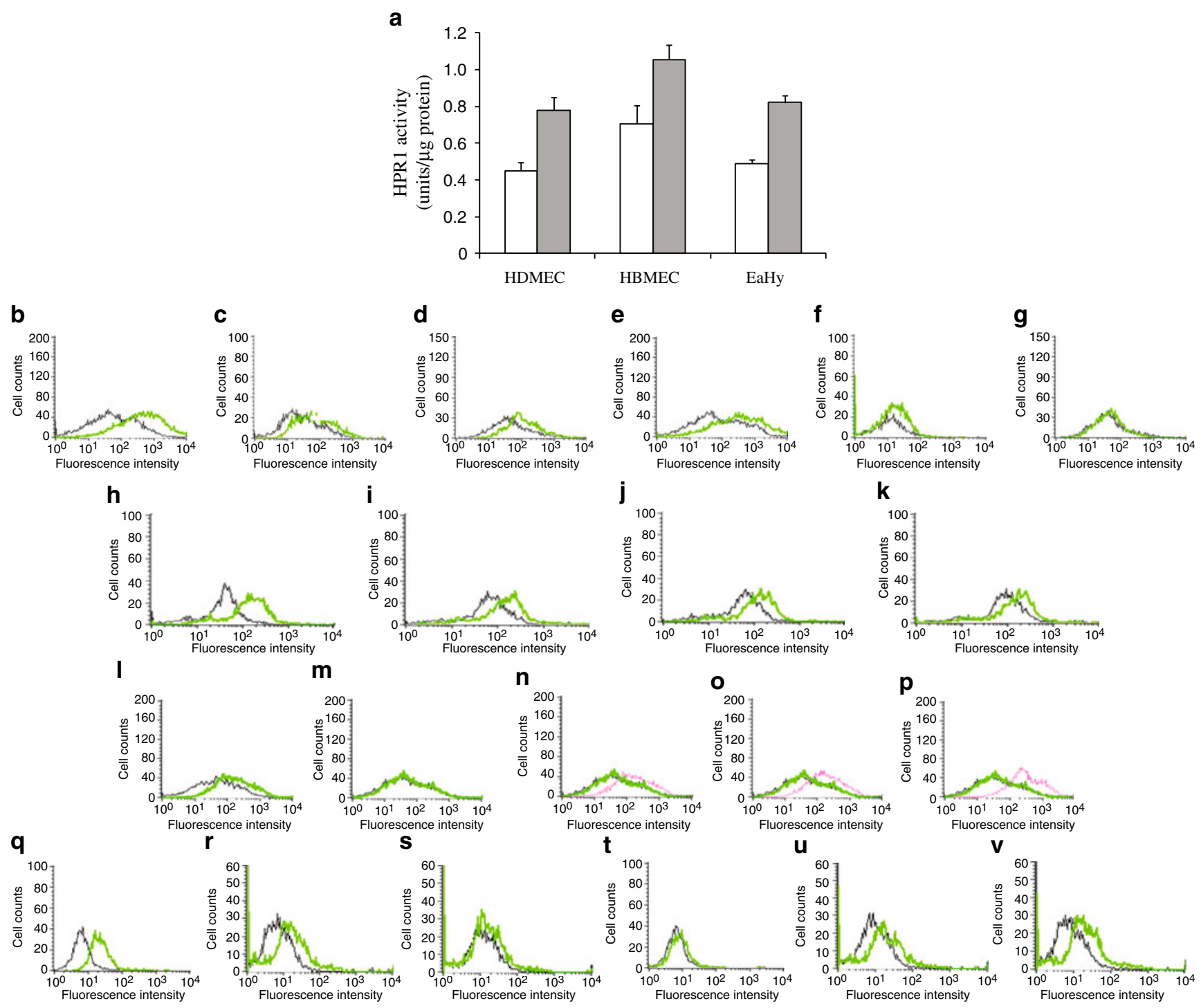

Fig. 1 High glucose increases HPR1 activity and decreases cell surface heparan sulphate in endothelial cells. a HDMEC, HBMEC and EaHy 926 cells were incubated in medium containing 5 (white bars) or $30 \mathrm{mmol} / \mathrm{l}$ (grey bars) glucose for $48 \mathrm{~h}$. Cells were then collected and analysed for HPR1 activity by ELISA. Data are mean \pm SD of three independent experiments. $\operatorname{HDMEC}(\mathbf{b}, \mathbf{e}), \operatorname{HBMEC}(\mathbf{c}, \mathbf{f})$ and EaHy $926(\mathbf{d}, \mathbf{g})$ cells incubated as above in medium containing $5(\mathbf{b}-\mathbf{d})$ or $30 \mathrm{mmol} / 1$ (e-g) glucose were also analysed for cell surface heparan sulphate levels by staining with an anti-heparan sulphate monoclonal antibody (green line) followed by FACS. Black line, mouse IgM used as isotype control. $\mathbf{h}-\mathbf{k}$ High glucose induces cell surface heparan sulphate loss in a dose-dependent manner. HBMEC were seeded overnight in a six-well plate in complete RPMI 1640 medium containing high glucose $(25 \mathrm{mmol} / \mathrm{l})$. Cells were then cultured under (h) 5, (i) 10, (j) 20 and (k) $30 \mathrm{mmol} / 1$ glucose and incubated for another $24 \mathrm{~h}$. Cells were collected and analysed for cell surface heparan sulphate levels by staining with a phage display-derived antibody (EW3D10) followed by flow cytometry. l-p Restoration of cell surface heparan sulphate levels by HPR1 inhibitors. HBMEC were incubated for $24 \mathrm{~h}$ in complete medium containing (I) 5 or (m) $30 \mathrm{mmol} / \mathrm{l}$ glucose in the absence or presence of (n) PI- 88 , (o) heparin or (p) sulodexide $(50 \mu \mathrm{g} / \mathrm{ml}$ each). Cell surface heparan sulphate was detected by FACS analysis of cells stained with anti-heparan sulphate monoclonal antibody. Black line, mouse IgM as isotype control; red line, HPR1 inhibitor; green line, anti-heparan sulphate monoclonal antibody in the absence of a HPR 1 inhibitor. $\mathbf{q}-\mathbf{v}$ Restoration of cell surface heparan sulphate levels by HPRI suppression. HBMEC at $5 \mathrm{mmol} / \mathrm{l}$ were either not transfected $(\mathbf{q})$ or transfected with $(\mathbf{r})$ pcDNA3.1 or (s) pcDNA3.1 at $30 \mathrm{mmol} / \mathrm{l}$, or transfected at $5 \mathrm{mmol} /$ 1 with (t) $\mathrm{pcDNA} / H P R 1$ and (u) $\mathrm{pcDNA} / H P R 1-\mathrm{AS}$, and (v) at $30 \mathrm{mmol} / 1$ with pcDNA/HPRl-AS. Transfection was by FuGENE6 transfection reagent. After incubation for $24 \mathrm{~h}$, the cells were treated with low or high glucose for $24 \mathrm{~h}$ and analysed for cell surface heparan sulphate levels by flow cytometry of EW3D10-stained cells 
a

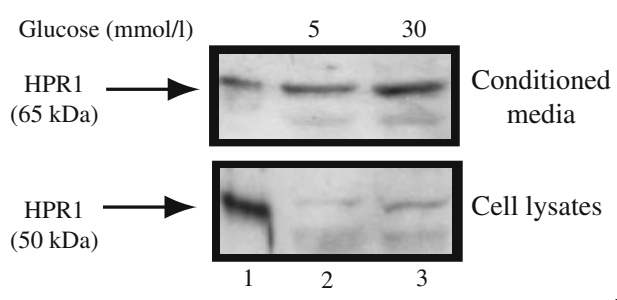

C

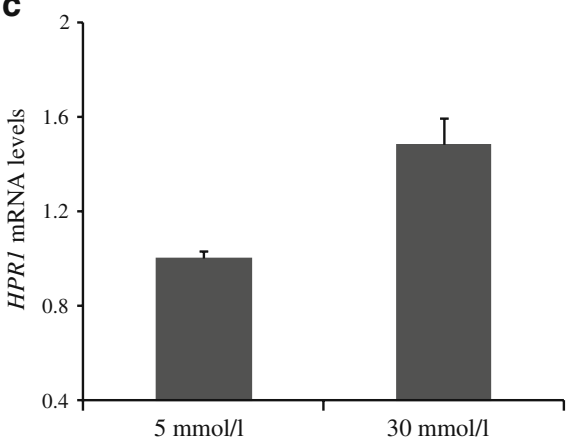

Fig. 2 High glucose induces HPR1 gene expression. a HPR1 protein in the supernatant fraction of HBMEC $(65 \mathrm{kDa})$ and in the cell lysates $(50 \mathrm{kDa})$ was analysed by Western blot with an anti-HPR1 rabbit IgG and an anti-HPR1 monoclonal antibody, respectively. Lane 1, purified platelet HPR1, was included as a positive control. b High glucose induces HPRl expression. HBMEC grown for $24 \mathrm{~h}$ in medium containing 5 or $30 \mathrm{mmol} / \mathrm{l}$ glucose were collected and analysed for HPRI mRNA by RT-PCR; semi-quantitative RT-PCR product was analysed in an agarose gel. c Quantification of real-time RT-PCR.

considered statistically significant. All statistics were conducted using SigmaStat3 software (Richmond, CA, USA).

\section{Results}

High glucose induces heparan sulphate degradation and HPR1 production Using a novel ELISA developed in our laboratory [29], we first showed that HPR1 activity in three endothelial cell lines, HDMEC, HBMEC and EaHy 926 cells, when cultured under high glucose conditions $(30 \mathrm{mmol} / \mathrm{l})$, was significantly increased by $73 \%(p=$ $0.005), 50 \%(p=0.023)$ and $67 \%(p=0.001$; Fig. 1a). Cell surface heparan sulphate levels in HDMEC under high glucose conditions ( $30 \mathrm{mmol} / \mathrm{l}$; Fig. 1e) were much lower than those under normal glucose levels ( $5 \mathrm{mmol} / \mathrm{l}$; Fig. $1 \mathrm{~b}$ ). Heparan sulphate was undetectable on the cell surface of HBMEC (Fig. 1f) and EaHy 926 cells (Fig. 1g) under high glucose conditions, but was present in the cells under normal glucose conditions (Fig. 1c, d). High glucose dosedependently induced the loss of cell surface heparan sulphate, as detected by EW3D10, a phage displayderived antibody that recognises sulphated heparan sulphate domains (Fig. 1h-k). b
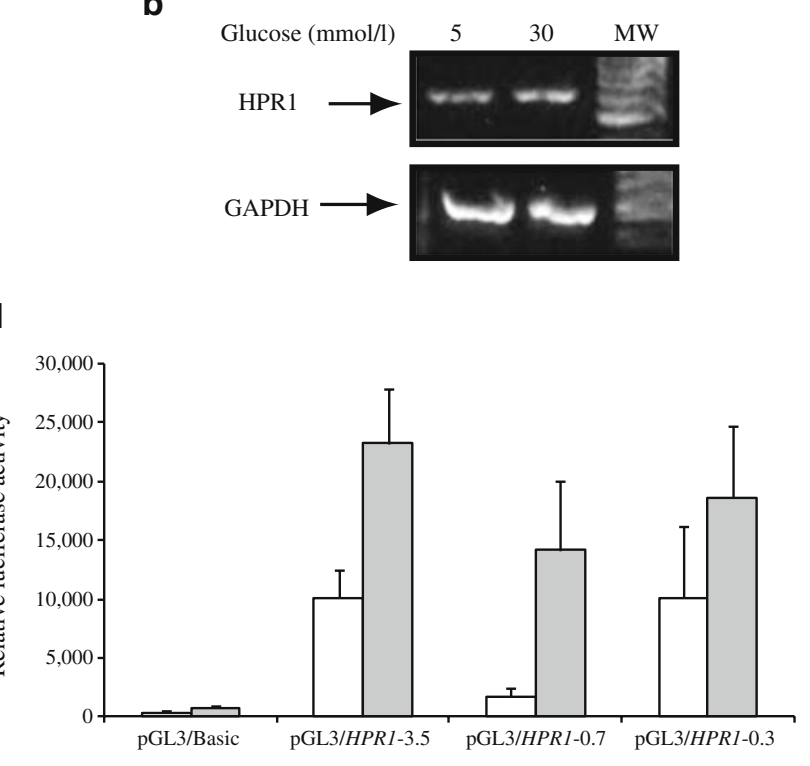

Data are mean \pm SD of a triplicate from one of three independent experiments with similar results. d HPR 1 promoter activity. HBMEC grown under normal (5 mmol/1; white bars) or high $(30 \mathrm{mmol} / 1$; grey bars) glucose were transfected with luciferase gene expression driven by the indicated lengths of the HPRI promoter. After incubation for $48 \mathrm{~h}$, cells were collected and analysed for luciferase activity. The results are mean $\pm \mathrm{SD}$ from one experiment in triplicate. The experiment was repeated twice with similar results

Three HPR1 inhibitors, PI-88, heparin and sulodexide $(50 \mu \mathrm{g} / \mathrm{ml}$ each) were able to restore cell surface heparan sulphate levels in HBMEC under high glucose conditions (Fig. 1n-p). Our previous study demonstrated that transfection of HT1080 cells with HPRI antisense gene (pcDNA/HPR1-AS) was able to increase cell surface heparan sulphate production [22]. Here, high glucose decreased cell surface heparan sulphate levels in pcDNA3.1-transfected (Fig. 1s), but not in pcDNA/HPR1AS-transfected HBMEC (Fig. 1v). Cell surface heparan sulphate was ablated in pcDNA/HPRl-transfected cells under normal glucose conditions (Fig. 1t).

To verify the ability of high glucose to induce HPR1 production, we first conducted Western blot to analyse HPR1 levels in the conditioned media and in the cytosol of HBMEC. As shown in Fig. 2a, HPR1 was detected as a $65 \mathrm{kDa}$ proenzyme in the supernatant fraction and mainly as a $50 \mathrm{kDa}$ protein in the cell lysates of HBMEC (Fig. 2a). HPR1 protein levels in conditioned media of HBMEC and in the cell lysates under high glucose conditions were much higher than those under normal glucose conditions (Fig. 2a).

RT-PCR revealed that HPR1 mRNA levels were higher in HBMEC under high than in those under normal glucose conditions (Fig. 2b). Real-time RT-PCR revealed a signif- 

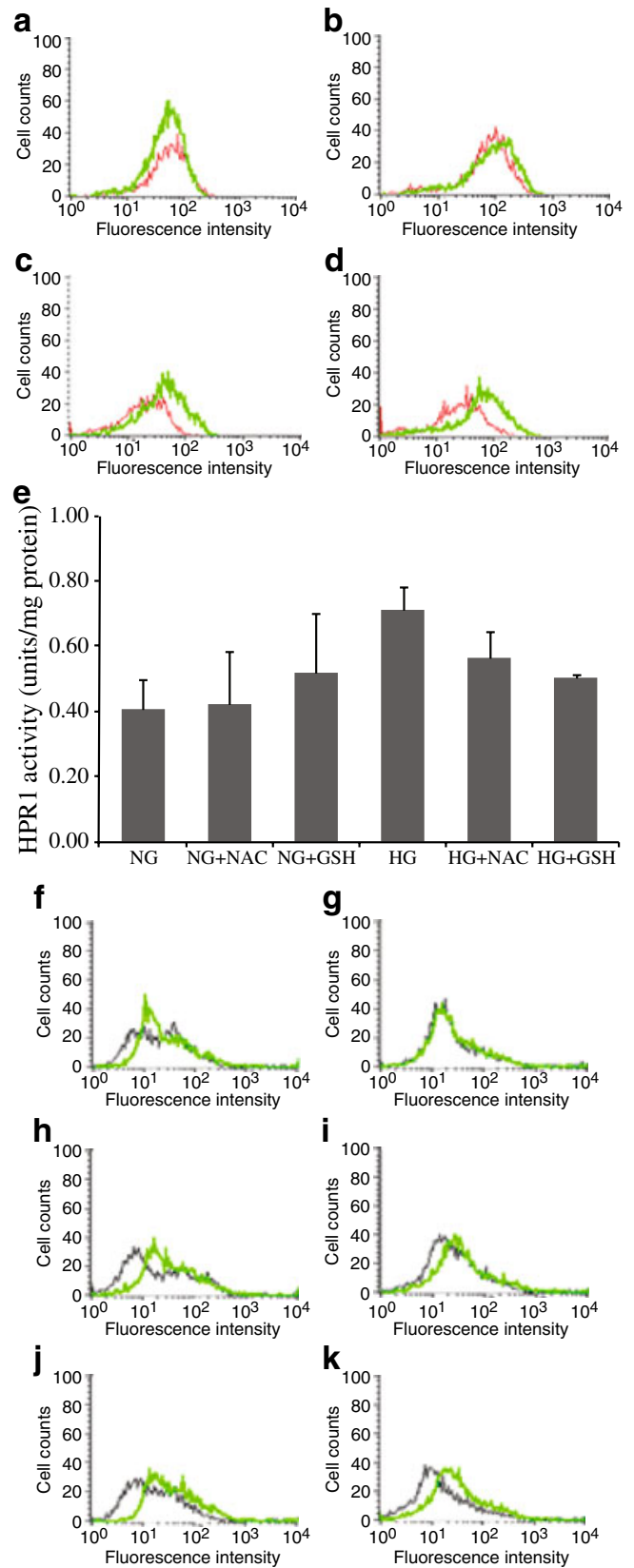

Fig. 3 High glucose-induced HPR1 production and heparan sulphate degradation is mediated by ROS. a-d Induction of ROS production in HBMEC by high glucose in a time-dependent manner. HBMEC were grown under high glucose conditions $(30 \mathrm{mmol} / \mathrm{l})$ for $0 \mathrm{~h} \mathrm{(a),} 24 \mathrm{~h} \mathrm{(b)}$, $48 \mathrm{~h}$ (c) and $96 \mathrm{~h}$ (d), and analysed for ROS production by FACS analysis of 2,7-dichlorodihydrofluorescin diacetate-loaded cells (green line). Red line, unloaded control. e HBMEC were grown for $48 \mathrm{~h}$ in normal glucose $(\mathrm{NG})$ or high glucose $(\mathrm{HG})$ conditions in the absence or presence of NAC or glutathione (GSH; $10 \mathrm{mmol} / \mathrm{l}$ each). Cells were collected and analysed for HPR1 activity by ELISA. Results are the mean \pm SD from one experiment in triplicate. The experiment was repeated twice with similar results. f-k HBMEC were treated as above (e) and cell surface heparan sulphate levels analysed by FACS. f Normal glucose $(5 \mathrm{mmol} / \mathrm{l})$, (g) high glucose $(30 \mathrm{mmol} / \mathrm{l})$, (h) normal glucose + NAC, (i) high glucose + NAC, (j) normal glucose + glutathione and (k) high glucose + glutathione. Black line, isotype control; green line, anti-heparan sulphate IgM icant increase of HPR1 mRNA levels by $49 \%(p<0.001$; Fig. 2c). HPR1 promoter-driven luciferase reporter assay revealed that luciferase gene expression driven by a 3.5-, 0.7- and 0.3-kb promoter in HBMEC under high glucose conditions was significantly higher than that under normal glucose conditions $(p=0.012, p=0.022$ and $p=0.019$ respectively; Fig. 2d). There was no significant difference in pGL3/Basic-transfected cells under normal or high glucose conditions $(p=0.053)$.

ROS production is required for high glucose-induced HPR1 production and heparan sulphate degradation ROS production is implicated in high glucose-induced HPR1 production in glomerular epithelial cells [19]. FACS analysis revealed that ROS production was increased in HBMEC under high glucose conditions in a time-dependent manner (Fig. 3a-d). HPR1 enzymatic activity assay revealed that HPR1 activity was increased by approximately $80 \%$ in HBMEC under high glucose conditions ( $p=$ 0.011 ; Fig. 3e), compared with that under normal glucose conditions. Two ROS scavengers, NAC and glutathione $(10 \mathrm{mmol} / \mathrm{l}$ each), were able to block high glucose-mediated increase of HPR 1 activity ( $p=0.024$ and $p=0.007$, respectively; Fig. 3b). Consistently, NAC and glutathione were able to restore cell surface heparan sulphate levels in high glucose-treated HBMEC (Fig. 3f-k).

$\mathrm{H}_{2} \mathrm{O}_{2}$ induces HPR1 production and heparan sulphate degradation Real-time RT-PCR revealed that HBMEC treated with exogenous $\mathrm{H}_{2} \mathrm{O}_{2}(1,5$ or $20 \mu \mathrm{mol} / 1)$ led to significantly increased HPRI mRNA transcription $(p<$ 0.001 for each concentration; Fig. 4a). $\mathrm{H}_{2} \mathrm{O}_{2}$ concentration at $1 \mu \mathrm{mol} / 1$ appeared to be slightly more effective than the two other concentrations in inducing HPR1 mRNA expression and production of $65 \mathrm{kDa}$ HPR1 protein in the supernatant fraction (Fig. 4b). Interestingly, $\mathrm{H}_{2} \mathrm{O}_{2}$ led to increased HPR1 production in the cytosol (Fig. 4b) and decreased cell surface heparan sulphate levels in a dosedependent manner (Fig. 4c-f). Decrease of cell surface heparan sulphate levels was blocked by PI-88, heparin and sulodexide (Fig. 4i-k).

Increased HPR1 production in endothelial cells of aortic arteries from a rat model of diabetes We analysed HPR 1 contents in the endothelial cells of aortic arteries from diabetic and normal rats. Immunohistochemistry staining with an anti-HPR1 monoclonal antibody on the cross sections of the aortic arteries revealed extensive HPR 1 presence in the endothelial cells and smooth muscle cells of the aortic arteries from two diabetic rats (Fig. 5c, d), but levels were low or not detectable in cells from two normal rats (Fig. 5a, b). We analysed HPR1 contents in the aortic arteries from six normal and five diabetic rats, and found 

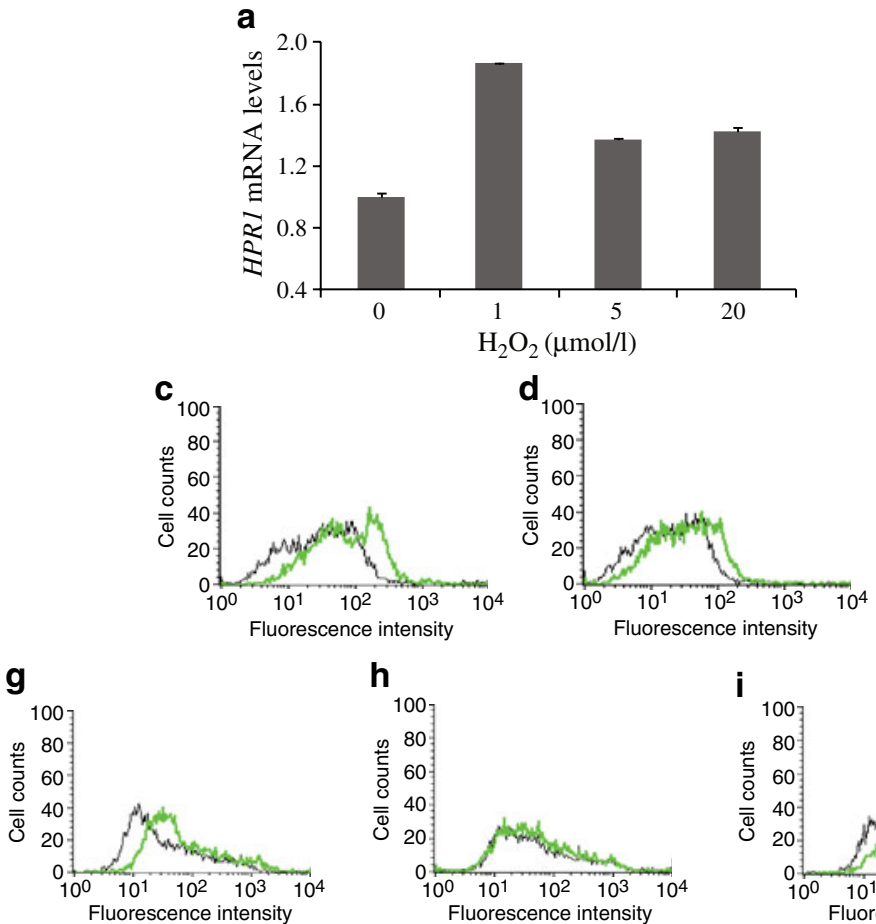

h

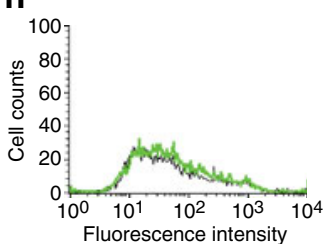

i

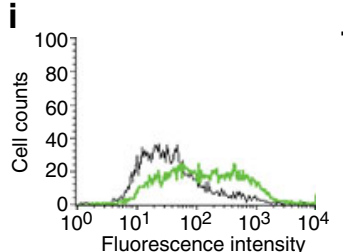

b

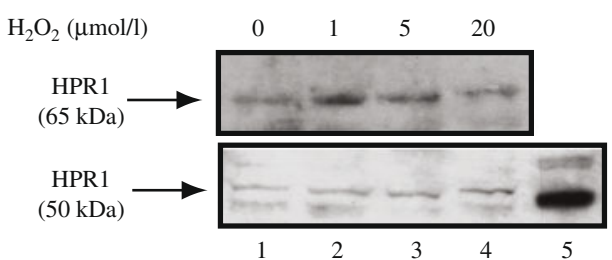

$\mathbf{e}_{100}$

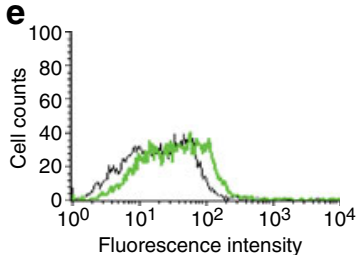

f
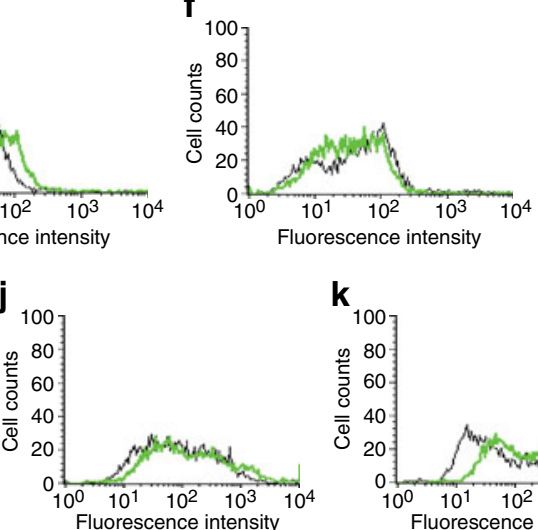

$\mathbf{k}$

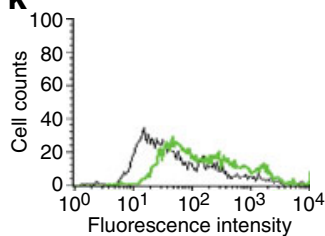

Fig. $4 \mathrm{H}_{2} \mathrm{O}_{2}$ induces HPR1 production and heparan sulphate degradation in HBMEC. HBMEC grown in medium containing $5 \mathrm{mmol} / \mathrm{l}$ glucose were stimulated with the indicated concentrations of $\mathrm{H}_{2} \mathrm{O}_{2}$. After incubation for $24 \mathrm{~h}$, the cells were collected. a Analysis of treated cells for HPR1 mRNA expression by real-time RT-PCR. Data represent the mean \pm SD of a triplicate from one of two experiments with similar results. b HPR 1 protein in conditioned medium and cytosol of HBMEC (cell lysate) was analysed by Western blot with antibodies against HPR1. Lane 5 shows a positive control of purified platelet HPR1. c-f Cell surface heparan sulphate levels by
FACS. HBMEC grown in normal glucose conditions were treated with $\mathrm{H}_{2} \mathrm{O}_{2}$ as follows: (c) $0 \mu \mathrm{mol} / \mathrm{l}$, (d) $1 \mu \mathrm{mol} / 1$, (e) $5 \mu \mathrm{mol} / 1$, (f) $20 \mu \mathrm{mol} / 1$. Cell surface heparan sulphate was analysed by FACS with an antiheparan sulphate monoclonal antibody. g-k HPR1 inhibitors block $\mathrm{H}_{2} \mathrm{O}_{2}$-induced heparan sulphate degradation. HBMEC grown in normal glucose conditions were stimulated for $24 \mathrm{~h}$ without $\mathrm{H}_{2} \mathrm{O}_{2}(\mathrm{~g})$ and with $\mathrm{H}_{2} \mathrm{O}_{2}$ in the absence (h) or presence of (i) PI-88, (j) heparin or $(\mathbf{k})$ sulodexide $(50 \mu \mathrm{g} / \mathrm{ml})$. Cells were collected and analysed for cell surface heparan sulphate levels. Black line, mouse IgM used as isotype control; green line, anti-heparan sulphate monoclonal antibody that HPR1 content in the endothelial cells of arteries of diabetic rats reached a significantly higher level than in control rats (Fig. 5e). HPR1 levels in smooth muscle cells were also increased.

HPR1 production in endothelial cells of atherosclerotic plaques We next tested whether HPR1 production was increased in arterial endothelial cells in hyperglycaemic patients. We first analysed HPR1 content in ten carotid artery autopsy specimens. HPR1 levels in endothelial cells and macrophages were graded as weakly positive in an autopsy specimen from a patient without diabetes and atherosclerosis (Fig. 6a). In contrast, HPR1 levels in endothelial cells and macrophages were graded as strongly positive in an autopsy specimen from a patient with diabetes and atherosclerosis (Fig. 6b). Normal mouse IgG was included as a negative control, revealing no nonspecific signal (Fig. 6c). Among seven non-diabetic autopsy-derived samples, HPR1 content in endothelial cells was graded as weakly positive in five specimens from patients without diabetes or severe atherosclerosis, but moderately positive or strongly positive in two patients, each with moderate or severe atherosclerosis (Table 1). Among autopsy-derived samples from three patients with diabetes and atherosclerosis, HPR1 content in endothelial cells was graded as strongly positive (Table 1). Macrophages were present in all specimens with moderate or severe atherosclerosis. HPR 1 was highly expressed in the macrophages of all specimens. HPR1 was also detectable in smooth muscle cells in many autopsy specimens (Table 1).

Preliminary analysis of these ten autopsy specimens suggests that increased HPR1 levels in endothelial cells are associated with hyperglycaemia and/or atherosclerotic lesions. To address this, we analysed HPR1 levels in endothelial cells in 15 carotid atherosclerotic lesions from six diabetic and nine non-diabetic living patients. HPR 1 content in endothelial cells was graded as minimal in an atherosclerotic lesion from a non-diabetic patient (Fig. 6d). However, in a carotid atherosclerotic lesion from a nondiabetic patient, HPR1 content was graded as strongly 

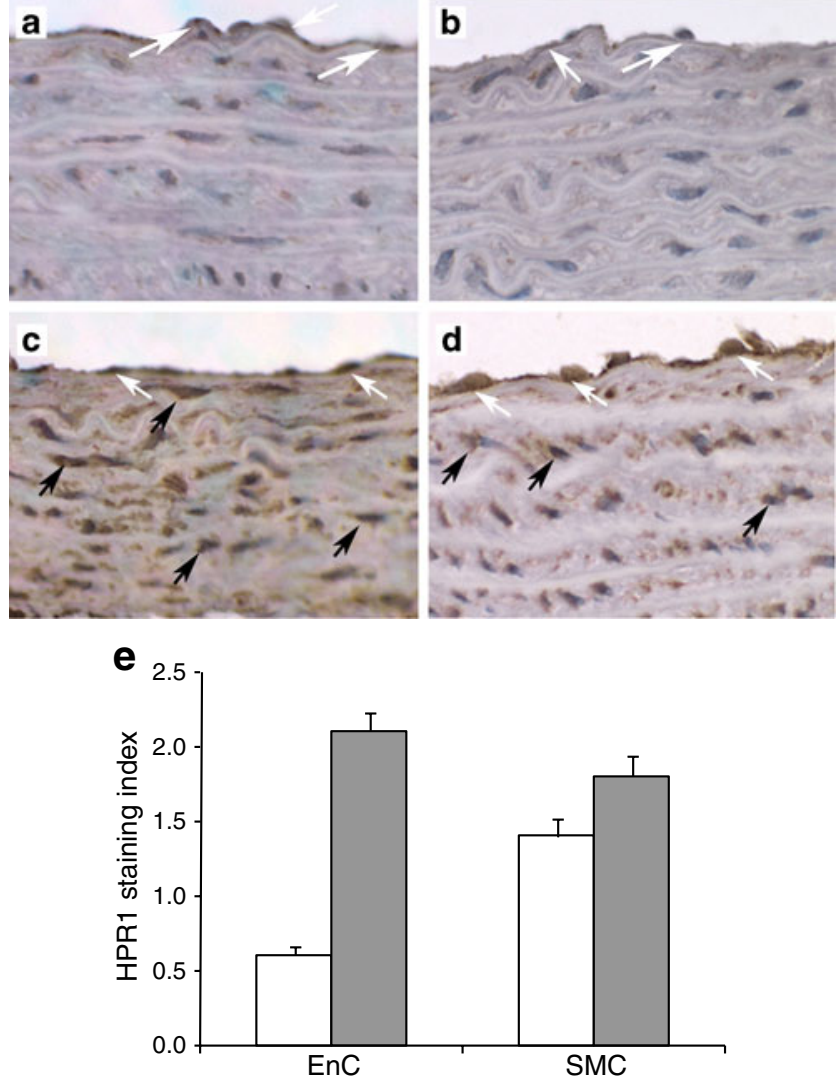

Fig. 5 Increased HPR1 production in the artery of diabetic rats. Aortic artery sections from normal $(\mathbf{a}, \mathbf{b})$ and diabetic $(\mathbf{c}, \mathbf{d})$ rats were stained with anti-HPR1 monoclonal antibody. White arrows, endothelial cells; black arrows, smooth muscle cells. e Increased HPR1 production in endothelial cells $(\mathrm{EnC})$ of aortic artery from diabetic rats (grey bars). Mean score of HPR1 positivity in endothelial cells of aortic artery from five diabetic rats was significantly higher than that in cells from six non-diabetic rats (white bars; $p=0.00086$ ). In contrast, mean score of HPR1 positivity in smooth muscle cells (SMC) from the same rats was not significantly different $(p=0.257)$

positive in endothelial cells and in infiltrating 'foam' cells (Fig. 6e). An example of where HPR1 levels in endothelial cells and macrophages in a specimen from a diabetic patient (Fig. 6f) were graded as strongly positive is also given.

Among six endarterectomy specimens from living nondiabetic patients, HPR1 positivity in endothelial cells was graded as negative and weakly positive (one each), and strongly positive in four samples (Table 2). HPR1 positivity in macrophages was graded as weakly positive in two, moderately positive in one and strongly positive in three samples (Table 2). Among nine endarterectomy specimens from diabetic patients, HPR1 positivity in endothelial cells was graded as negative in two, weakly positive in one and strongly positive in six specimens (Table 2). HPR1 positivity in macrophages was graded as weakly positive in two, moderate in one and strongly positive in six samples (Table 2). There was no statistical difference in HPR1 positivity in endothelial cells or in macrophages in atherosclerotic lesions from living patients with or without diabetes $(p>0.05)$.

\section{Discussion}

Our present study has demonstrated that HPR1 activity in a hybrid (EaHy 926) and in two microvascular endothelial cell lines (HBMEC, HDMEC) under high glucose conditions was increased. Increased HPR1 promoter activity and HPR1 mRNA levels (Fig. 2) in HBMEC under high glucose conditions suggests a mechanism affecting transcriptional regulation of HPRI expression. It should be noted that differences in response to high glucose stimulation are possible between primary endothelial cells and immortalised endothelial cell lines, and between endothelial cells derived from micro- and macrovascular vessels. Nevertheless, a recent study showed that HPRI mRNA levels and HPR 1 activity were increased in primary porcine aortic endothelial cells under high glucose conditions [20]. Our study further showed that increased HPR1 production led to decreased cell surface heparan sulphate levels, and that HPR1 inhibitors and HPR 1 suppression by HRP1 antisense transfection restored levels of cell surface heparan sulphate levels. Taken together, these observations suggest that increased HPR1 production under hyperglycaemic conditions is responsible for decreased cell surface heparan sulphate levels in endothelial cells. The causal role of HPR1 in degrading cell surface heparan sulphate was confirmed by showing the loss of cell surface heparan sulphate in HPR1-transfected HBMEC (Fig. 1t).

We noticed that induction of HPR 1 production by high glucose in endothelial cells was only moderate. High glucose led to increased HPRI gene expression and HPR1 activity in lysates of HBMEC by about $50 \%$. However, this level of increased HPR1 production may have a much more profound effect on heparan sulphate degradation, as HPR1 enzyme can repeatedly cleave heparan sulphate. In addition, high glucose may also increase secretion of HPR1 after processing in the lysosome of endothelial cells [35]. Therefore, high glucose may have a dual effect on HPR1 production and secretion. This notion is supported by our finding that high glucose $(30 \mathrm{mmol} / \mathrm{l})$ led to loss of heparan sulphate on the cell surface in three endothelial cell lines (Fig. 1e-g). The physiological relevance of this observation was confirmed by showing that high glucose induced loss of cell surface heparan sulphate in a dose-dependent manner. High glucose $(10 \mathrm{mmol} / \mathrm{l})$ was able to significantly decrease cell surface heparan sulphate levels (Fig. 1i).

ROS have been implicated as critical 'players' in the regulation of HPR1 expression in different model systems. 

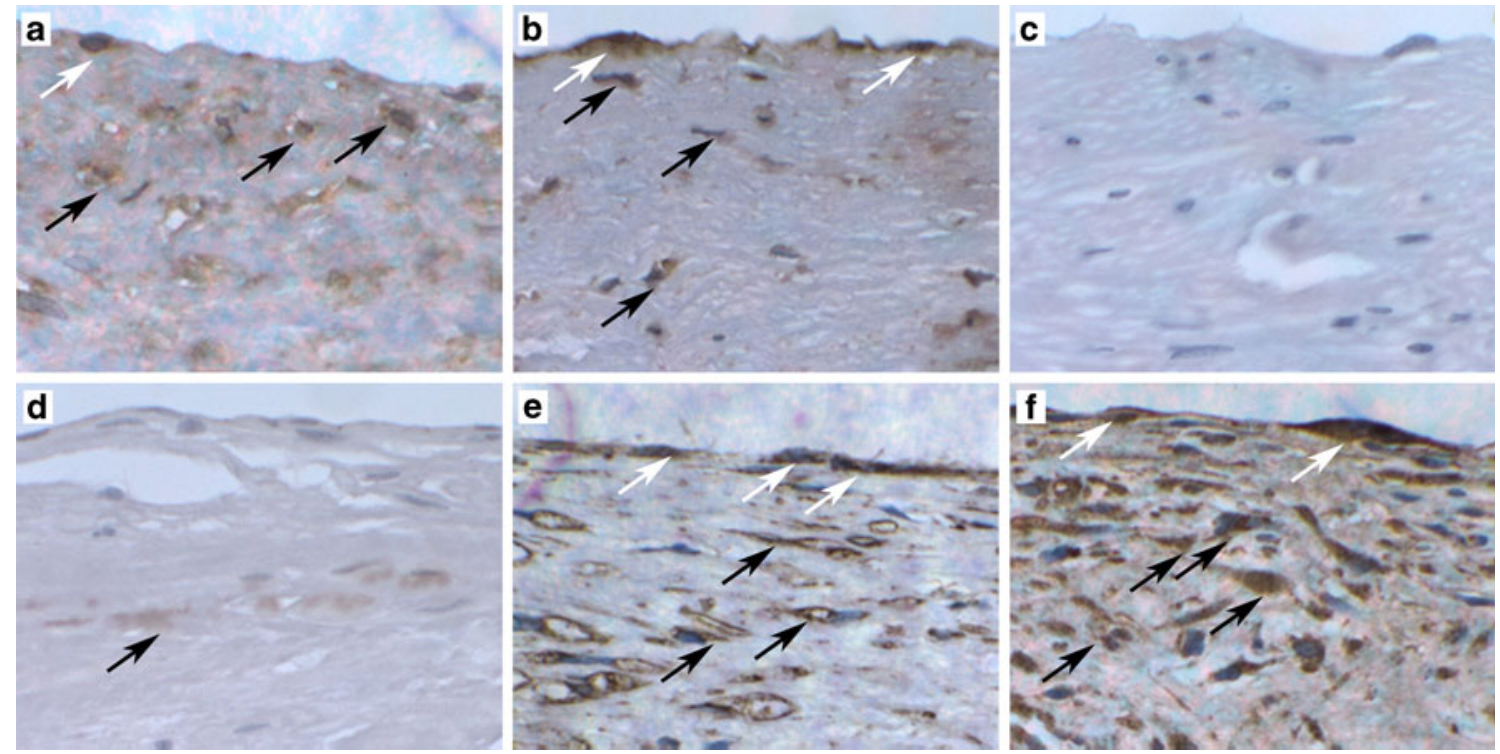

Fig. 6 Increased HPR1 content in endothelial cells of carotid arterial walls from patients with diabetes and/or atherosclerosis. Crosssections of carotid artery from autopsy-derived specimens $(\mathbf{a}-\mathbf{c})$ were stained with anti-HPR1 monoclonal antibody. a Carotid artery from a non-diabetic patient with minimal atherosclerosis (patient 3, Table 1). b Carotid artery from a diabetic patient with severe atherosclerosis (patient 8, Table 1). c No non-specific signal with normal mouse IgG as negative control on a cross-section of carotid artery from a non- diabetic patient with myocardial infarction. d-f Cross-sections of carotid atherosclerotic lesions from surgical specimens as follows: carotid artery (d) from a non-diabetic patient (patient 1, Table 2), (e) from a non-diabetic patient with severe atherosclerosis (patient 3, Table 2) and (f) from a diabetic patient with severe atherosclerosis (patient 15, Table 2). White arrows, endothelial cells; black arrows, 'foam cells' or macrophages
For example, ROS production is required for adriamycininduced HPR1 production in glomerular epithelial cells [19]. The role of ROS in regulating HPR1 expression in glomerular epithelial cells was further confirmed in vitro [36]. A recent study showed that ROS scavenging by overabundance of extracellular superoxide dismutase leads to decreased HPR1 production and increased cell surface heparan sulphate levels in a breast cancer cell line [37]. In the present study, we identified ROS as a critical component in mediating high glucose-induced HPR1 production. This was supported by the finding that: (1) ROS levels were increased in HBMEC under high glucose conditions; (2) glutathione and NAC, two ROS scavengers, were able to block high glucose-induced HPR1 production and heparan sulphate degradation; and (3) exogenous ROS were able to induce HPR1 production and heparan sulphate degradation. Together, these observations suggest that ROS play a critical role in mediating high glucose-induced HPR1 production. It should be noted that ROS are also capable of directly shedding off heparan sulphate side chains by heparan sulphate depolymerisation [38, 39]. Our experiment in Fig. 3 showed that ROS at higher concentrations led to complete loss of heparan sulphate on the cell surface, but did not proportionately increase HPR 1 production in conditioned media or HPRI gene expression. It is possible that ROS may reduce cell surface heparan sulphate levels by inducing HPR1 production and by depolymerising heparan sulphate.

It has been well established that ROS production is increased in endothelial cells under hyperglycaemic conditions [40]. Our present study demonstrated that HPR1 production was increased in endothelial cells of aortic arteries of diabetic rats. Though ROS production was not measured in vivo, we speculate that increased ROS production in endothelial cells under hyperglycaemic conditions plays a critical role in inducing HPR1 production. Consistently, HPR 1 production was also increased in glomerular endothelial cells in an adriamycin-induced model of nephropathy, due to increased ROS production [19]. In addition, we found that HPR1 production was very low in the endothelial cells of six autopsy-derived carotid artery specimens from non-diabetic individuals whose arteries were normal. However, there was strong HPR1 positivity in the autopsy-derived endothelial cells of carotid artery from three patients with diabetes and atherosclerosis, as well as in those from one patient without diabetes but with atherosclerosis.

Our animal experiment revealed that HPR1 levels were increased in endothelial cells. While our animal and clinical studies together suggest that hyperglycaemia could lead to increased HPR1 production in endothelial cells in vivo, this notion should be treated with caution 
as our study has several limitations. First, the numbers of samples in our study were very small. Second, nondiabetic control specimens were obtained from patients with a variety of diseases. Third, streptozotocin, as used to induce diabetes, may also induce ROS production. And finally, the duration of diabetes in rats prior to death was very short. Clearly, this may not reflect the situation in diabetic patients undergoing hyperglycaemia for a very long time.

In addition to hyperglycaemia, the inflammatory environment in atherosclerotic lesions may further enhance HPR1 production in endothelial cells. For example, HPR1 is produced locally by the endothelium at the site of delayed-type hypersensitivity-associated inflammation, and TNF- $\alpha$ and IFN- $\gamma$ can induce HPR1 production in cultured endothelial cells [41]. TNF- $\alpha$, IL-1 $\beta$ and fatty acids are able to induce HPR1 production in endothelial cells in vitro [42]. Immunohistochemical analyses of cross sections of aorta revealed intense staining for HPR 1 in the endothelium of Apoe-null mice but not in that of wild-type mice [42]. In our study, we found that HRP1 was highly abundant in endothelial cells in atherosclerotic plaques from nondiabetic patients. These observations suggest that many pathogenic factors in the atherosclerotic plaque can contribute to increased HPR1 production in the endothelial cells.

Accumulating evidence indicates that downregulation of HSPG under hyperglycaemic conditions plays an important role in the pathogenesis of atherogenesis [10]. HPR1 is involved in remodelling of the arterial structure, mechanics and repair in an endovascular stenting model [43]. Hprl overexpression in Hprl transgenic mice leads to increased arterial thickness, cellular density and mechanical compliance [43]. Moreover, HPR1 production is increased in the neointima of obese, hyperlipidaemic rats with endovascular stenting, compared with lean rats [43]. HPR1 levels in the neointima strongly correlate with neointimal thickness [43]. HPR1 production was also increased in the coronary artery of diabetic, hyperlipidaemic swine, mostly in macrophages enriched in the atherosclerotic lesions [44]. Our present study has demonstrated that HPR1 production was increased in the artery of diabetic rats. Our clinical study demonstrated that HPR 1 protein was highly detected in the 'foam' cells of atherosclerotic lesions, confirming the observation made in a recent study, which showed that HPR1 production was increased in macrophages in atherosclerotic lesions from three dialysis patients [45]. These observations suggest that increased HPR1 production in endothelial cells and macrophages is responsible for decreased amounts of HSPG in the arterial wall, and that increased HPR 1 production may contribute to the pathogenesis of atherosclerosis.
Acknowledgements This work was supported in part by the University Council of Research of Rush University Medical Center (to X. Xu). We thank J. Platt (Department of Surgery, University of Michigan, Ann Arbor MI, USA) for kindly providing the purified platelet heparanase and H.-Q. Miao (ImClone Systems) for kindly providing anti-HPR1 antibodies. We are also very thankful to Progen Pharmaceuticals for kindly providing PI- 88 and to R. Niecestro at Keryx Biopharmaceutics (New York, NY, USA) for kindly providing sulodexide.

Duality of interest The authors declare that there is no duality of interest associated with this manuscript.

\section{References}

1. Esko JD, Lindahl U (2001) Molecular diversity of heparan sulfate. J Clin Invest 108:169-173

2. Iozzo RV (2001) Heparan sulfate proteoglycans: intricate molecules with intriguing functions. J Clin Invest 108:165-167

3. Perrimon N, Bernfield M (2000) Specificities of heparan sulphate proteoglycans in developmental processes. Nature 404:725-728

4. Stringer SE, Gallagher JT (1997) Specific binding of the chemokine platelet factor 4 to heparan sulfate. J Biol Chem 272:20508-20514

5. Stringer SE, Gallagher JT (1997) Heparan sulphate. Int J Biochem Cell Biol 29:709-714

6. Gallagher JT (2001) Heparan sulfate: growth control with a restricted sequence menu. J Clin Invest 108:357-361

7. Iozzo RV, Cohen IR, Grassel S, Murdoch AD (1994) The biology of perlecan: the multifaceted heparan sulphate proteoglycan of basement membranes and pericellular matrices. Biochem J 302:625-639

8. Duan W, Paka L, Pillarisetti S (2005) Distinct effects of glucose and glucosamine on vascular endothelial and smooth muscle cells: evidence for a protective role for glucosamine in atherosclerosis. Cardiovasc Diabetol 4:16

9. Vogl-Willis CA, Edwards IJ (2004) High-glucose-induced structural changes in the heparan sulfate proteoglycan, perlecan, of cultured human aortic endothelial cells. Biochim Biophys Acta 1672:36-45

10. Wasty F, Alavi MZ, Moore S (1993) Distribution of glycosaminoglycans in the intima of human aortas: changes in atherosclerosis and diabetes mellitus. Diabetologia 36:316-322

11. Edwards IJ, Wagner JD, Vogl-Willis CA, Litwak KN, Cefalu WT (2004) Arterial heparan sulfate is negatively associated with hyperglycemia and atherosclerosis in diabetic monkeys. Cardiovasc Diabetol 3:6

12. Pillarisetti S (2000) Lipoprotein modulation of subendothelial heparan sulfate proteoglycans (perlecan) and atherogenicity. Trends Cardiovasc Med 10:60-65

13. Olsson U, Ostergren-Lunden G, Moses J (2001) Glycosaminoglycan-lipoprotein interaction. Glycoconj J 18:789-797

14. Conde-Knape K (2001) Heparan sulfate proteoglycans in experimental models of diabetes: a role for perlecan in diabetes complications. Diabetes Metab Res Rev 17:412-421

15. Pillarisetti S, Paka L, Obunike JC, Berglund L, Goldberg IJ (1997) Subendothelial retention of lipoprotein (a). Evidence that reduced heparan sulfate promotes lipoprotein binding to subendothelial matrix. J Clin Invest 100:867-874

16. van den Hoven MJ, Wijnhoven TJ, Li JP et al (2008) Reduction of anionic sites in the glomerular basement membrane by heparanase does not lead to proteinuria. Kidney Int 73:278-287

17. van den Hoven MJ, Rops AL, Bakker MA et al (2006) Increased expression of heparanase in overt diabetic nephropathy. Kidney Int 70:2100-2108 
18. Maxhimer JB, Somenek M, Rao G et al (2005) Heparanase-1 gene expression and regulation by high glucose in renal epithelial cells: a potential role in the pathogenesis of proteinuria in diabetic patients. Diabetes 54:2172-2178

19. Kramer A, van den Hoven M, Rops A et al (2006) Induction of glomerular heparanase expression in rats with adriamycin nephropathy is regulated by reactive oxygen species and the reninangiotensin system. J Am Soc Nephrol 17:2513-2520

20. Han J, Woytowich AE, Mandal AK, Hiebert LM (2007) Heparanase upregulation in high glucose-treated endothelial cells is prevented by insulin and heparin. Exp Biol Med (Maywood) 232:927-934

21. Lum H, Qiao J, Walter RJ et al (2003) Inflammatory stress increases receptor for lysophosphatidylcholine in human microvascular endothelial cells. Am J Physiol Heart Circ Physiol 285:H1786-H1789

22. Xu X, Rao G, Quiros RM et al (2007) In vivo and in vitro degradation of heparan sulfate (HS) proteoglycans by HPR1 in pancreatic adenocarcinomas. Loss of cell surface HS suppresses fibroblast growth factor 2-mediated cell signaling and proliferation. J Biol Chem 282:2363-2373

23. Wijnhoven TJ, van den Hoven MJ, Ding H et al (2008) Heparanase induces a differential loss of heparan sulphate domains in overt diabetic nephropathy. Diabetologia 51:372-382

24. Xu X, Ding J, Rao G et al (2007) Estradiol induces heparanase-1 expression and heparan sulphate proteoglycan degradation in human endometrium. Hum Reprod 22:927-937

25. Quiros RM, Rao G, Plate J et al (2006) Elevated serum heparanase-1 levels in patients with pancreatic carcinoma are associated with poor survival. Cancer 106:532-540

26. Jiang P, Kumar A, Parrillo JE et al (2002) Cloning and characterization of the human heparanase-1 (HPR1) gene promoter: role of GA-binding protein and $\mathrm{Sp} 1$ in regulating HPR1 basal promoter activity. J Biol Chem 277:8989-8998

27. Eustice DC, Feldman PA, Colberg-Poley AM, Buckery RM, Neubauer RH (1991) A sensitive method for the detection of betagalactosidase in transfected mammalian cells. Biotechniques 11 (739-740):742-743

28. Francis DJ, Parish CR, McGarry M et al (2003) Blockade of vascular smooth muscle cell proliferation and intimal thickening after balloon injury by the sulfated oligosaccharide PI-88: phospHomannopentaose sulfate directly binds FGF-2, blocks cellular signaling, and inhibits proliferation. Circ Res 92:e70-e77

29. Xu X, Quiros RM, Maxhimer JB et al (2003) Inverse correlation between heparan sulfate deposition and heparanase-1 gene expression in thyroid papillary carcinomas: a potential role in tumor metastasis. Clin Cancer Res 9:5968-5979

30. Rao G, Liu D, Xing M, Tauler J, Prinz RA, Xu X (2010) Induction of heparanase-1 expression by mutant $b$-raf kinase: role of GA binding protein in heparanase-1 promoter activation. Neoplasia 12:946-956
31. Abboud-Jarrous G, Rangini-Guetta Z, Aingorn H et al (2005) Site-directed mutagenesis, proteolytic cleavage and activation. J Biol Chem 280:13568-13575

32. Zetser A, Levy-Adam F, Kaplan V et al (2004) Processing and activation of latent heparanase occurs in lysosomes. J Cell Sci $117: 2249-2258$

33. Nadav L, Eldor A, Yacoby-Zeevi O et al (2002) Activation, processing and trafficking of extracellular heparanase by primary human fibroblasts. J Cell Sci 115:2179-2187

34. Maxhimer JB, Quiros RM, Stewart R et al (2002) Heparanase-1 expression is associated with the metastatic potential of breast cancer. Surgery 132:326-333

35. Wang F, Kim MS, Puthanveetil P et al (2009) Endothelial heparanase secretion after acute hypoinsulinemia is regulated by glucose and fatty acid. Am J Physiol Heart Circ Physiol 296: H1108-H1116

36. van den Hoven MJ, Waanders F, Rops AL et al (2009) Regulation of glomerular heparanase expression by aldosterone, angiotensin II and reactive oxygen species. Nephrol Dial Transplant 24:2637-2645

37. Teoh ML, Fitzgerald MP, Oberley LW, Domann FE (2009) Overexpression of extracellular superoxide dismutase attenuates heparanase expression and inhibits breast carcinoma cell growth and invasion. Cancer Res 69:6355-6363

38. Raats CJ, van Den Born J, Berden JH (2000) Glomerular heparan sulfate alterations: mechanisms and relevance for proteinuria. Kidney Int 57:385-400

39. Moseley R, Waddington R, Evans P, Halliwell B, Embery G (1995) The chemical modification of glycosaminoglycan structure by oxygen-derived species in vitro. Biochim Biophys Acta 1244:245-252

40. Stocker R, Keaney JF Jr (2004) Role of oxidative modifications in atherosclerosis. Physiol Rev 84:1381-1478

41. Edovitsky E, Lerner I, Zcharia E, Peretz T, Vlodavsky I, Elkin M (2006) Role of endothelial heparanase in delayed-type hypersensitivity. Blood 107:3609-3616

42. Chen G, Wang D, Vikramadithyan R et al (2004) Inflammatory cytokines and fatty acids regulate endothelial cell heparanase expression. Biochemistry 43:4971-4977

43. Baker AB, Groothuis A, Jonas M et al (2009) Heparanase alters arterial structure, mechanics, and repair following endovascular stenting in mice. Circ Res 104:380-387

44. Baker AB, Chatzizisis YS, Beigel R et al (2010) Regulation of heparanase expression in coronary artery disease in diabetic, hyperlipidemic swine. Atherosclerosis 213:436-442

45. Cohen-Mazor M, Sela S, Mazor R et al (2008) Are primed polymorphonuclear leukocytes contributors to the high heparanase levels in hemodialysis patients? Am J Physiol Heart Circ Physiol 294:H651-H658 